\title{
Does N-terminal Pro-brain Type Natriuretic Peptide Predict Cardiac Complications After Hip Fracture Surgery?
}

\author{
Hiroki Ushirozako MD, Tsuyoshi Ohishi MD, PhD, Tomotada Fujita MD, \\ Daisuke Suzuki MD, PhD, Kazufumi Yamamoto MD, PhD, Tomohiro Banno MD, \\ Hiroyuki Takase MD, PhD, Yukihiro Matsuyama MD, PhD
}

Received: 30 September 2016/Accepted: 11 January 2017/Published online: 18 January 2017

(C) The Association of Bone and Joint Surgeons ® 2017

\begin{abstract}
Background Elderly patients with hip fracture are at risk for cardiac complications. N-terminal pro-brain type natriuretic peptide (NT-proBNP) has been shown to predict cardiac complications in surgical patients; however, to our

Each author certifies that he or she, or a member of their immediate family, has no commercial associations (eg, consultancies, stock ownership, equity interest, patent/licensing arrangements, etc) that might pose a conflict of interest in connection with the submitted article.

All ICMJE Conflict of Interest Forms for authors and Clinical Orthopaedics and Related Research ${ }^{\mathbb{R}}$ editors and board members are on file with the publication and can be viewed on request. Each author certifies that his or her institution has approved the human protocol for this investigation, that all investigations were conducted in conformity with ethical principles of research, and that informed consent was obtained.

This work was performed at Enshu Hospital, Hamamatsu, Shizuoka, Japan.
\end{abstract}

H. Ushirozako ( $\square)$

Department of Orthopaedic Surgery, Fujinomiya City Hospital, 3-1 Nishiki-Cho, Fujinomiya, Shizuoka 418-0076, Japan e-mail: verisa0808@gmail.com

T. Ohishi, T. Fujita, D. Suzuki

Department of Orthopaedic Surgery, Enshu Hospital,

Hamamatsu, Shizuoka, Japan

K. Yamamoto

Department of Orthopaedic Surgery, Shintoshi Hospital, Iwata, Shizuoka, Japan

T. Banno, Y. Matsuyama

Department of Orthopaedic Surgery, Hamamatsu University

School of Medicine, Higashi-Ku, Hamamatsu, Shizuoka, Japan

H. Takase

Department of Internal Medicine, Enshu Hospital, Naka-Ku, Hamamatsu, Shizuoka, Japan knowledge, only two studies have evaluated the utility of this test in patients with hip fracture. We believe it is important to assess a more accurate cutoff value of NTproBNP with exclusion of patients with renal failure.

Questions/Purposes To assess the association between preoperative NT-proBNP and cardiac complications after hip fracture surgery.

Methods We performed 450 surgical procedures in patients with hip fractures between January 2011 and December 2014. Exclusion criteria were renal dysfunction and inadequate laboratory tests. The final study population consisted of 328 patients (mean age, 83 years; 80\% women). Preoperatively, measurement of NT-proBNP level was performed. The primary endpoint was the occurrence of cardiac complications within 14 days after surgery based on a chart review. The predictive value of NT-proBNP was assessed using multivariate logistic regression analysis, controlling for relevant confounding variables such as age, gender, body weight, and renal function; we also performed receiver operating characteristic (ROC) curve analysis. Postoperative cardiac complications were encountered in $7 \%$ of patients ( 24 of 328).

Results The median preoperative NT-proBNP level was higher in patients with complications than in those without (1090 [interquartile range, $614-3191 \mathrm{pg} / \mathrm{mL}$ ] vs $283 \mathrm{pg} /$ $\mathrm{mL}$ [interquartile range, $137-507 \mathrm{pg} / \mathrm{mL}$ ], $\mathrm{p}<0.001$ ). The cutoff level of NT-proBNP determined by ROC curve analysis was $600 \mathrm{pg} / \mathrm{mL}$, with a sensitivity, specificity, positive predictive value, and negative predictive value of $79 \%, 81 \%, 25 \%$, and $98 \%$, respectively, and the area under the ROC curve was 0.87 (95\% CI, 0.80-0.94; p < 0.001). After controlling for potentially relevant confounding variables, we found a preoperative NT-proBNP greater than $600 \mathrm{pg} / \mathrm{mL}$ was associated with an increased risk of 
cardiac complications (odds ratio, 13; 95\% CI, 4-38; $\mathrm{p}<0.001)$ compared with those with NT-proBNP less than $600 \mathrm{pg} / \mathrm{mL}$.

Conclusions Preoperative NT-proBNP greater than $600 \mathrm{pg} / \mathrm{mL}$ is independently associated with postoperative cardiac complications in patients with hip fracture without renal dysfunction. NT-proBNP measurement provides additional information and is clinically useful for predicting cardiac complications during the early phase after hip fracture surgery. Future studies might develop a simple index for prediction of postoperative cardiac complication including cutoff values of NT-proBNP.

Level of Evidence Level III, diagnostic study.

\section{Introduction}

In Japan, nearly 150,000 patients experienced a hip fracture in 2007, and one prediction suggested this number is likely to increase to 250,000 by 2020 [13]. Hip fracture surgery is associated with high in-hospital mortality $(1.38 \%$; mean length of stay 35 days) and frequent cardiac complications $(1.07 \%)$ [9], so identification of patients at risk for postoperative cardiac events is crucial $[2,12,16]$. Before and after surgery, routine conventional clinical assessments often are performed, but they often are insufficient. Patients with identifiable risk factors for cardiac disease may undergo noninvasive examinations for ischemia and cardiac function; however, these methods involve high healthcare costs, and can be time consuming. Therefore, it is desirable to identify patients at risk for postoperative cardiac complications more easily, accurately, and less expensively.

Several tools can help clinicians anticipate complications after hip fracture surgery, including the American Society of Anesthesiologists (ASA) classification [18], American Heart Association (AHA) guidelines [5], Goldman Cardiac Risk Index (GCRI) [7], and Revised Cardiac Risk Index (RCRI) [10]. Although those evaluation tools cost nothing to apply and are relatively accurate, many orthopaedic surgeons are not familiar with the most-useful ones on that list (the AHA guidelines, the GCRI, and the RCRI), and those may need technical examinations such as auscultation by cardiologists or internists; they also are not completely accurate. To date, there have been two reports for the role of N-terminal probrain type natriuretic peptide (NT-proBNP) as a predictive factor for postoperative cardiac complications or mortalities in patients with hip fractures [12, 14]. An earlier small study on orthopaedic patients found preoperative B-type natriuretic peptide (BNP) elevation to be superior to the ASA classification in independently predicting postoperative cardiac complications [19].
However, those studies also included patients with hip fractures who have renal dysfunction, even though NTproBNP level is known to be strongly affected by renal function [17].

We therefore wished to assess the association between preoperative NT-proBNP and cardiac complications during the immediate postoperative period after hip fracture surgery.

\section{Patients and Methods}

Study Design and Setting

This was an observational followup study to assess the association between preoperative NT-proBNP and cardiac complications during the first 2 weeks after hip fracture surgery. The study complied with the principles of the Declaration of Helsinki, and the study protocol was approved by the Ethics Committee of Enshu Hospital. All participants gave written informed consent to participate before the start of the study.

\section{Participants/Study Subjects}

Between January 2011 and December 2014, we performed 450 surgical procedures in patients with hip fractures. Of those, 439 (98\%) underwent measurement of NT-proBNP; during this time, we generally used the test for all patients with hip fractures with planned surgery. We generally did not use the test when informed consent for surgery or measuring NT-proBNP could not be obtained from the patient or patient's relatives. All 439 (100\%) patients had complete datasets including surgical data and postoperative records sufficient for analysis. Patients with renal dysfunction (creatinine clearance $<30 \mathrm{~mL} /$ minute; estimated by the Cockcroft and Gault method [4]) were excluded because renal function is known to strongly affect NT-proBNP level [17]; the final study population consisted of 328 patients with hip fractures. Most of the patients were female (263 of 328; 80\%), and the mean age was 83 years (range, 32-98 years). During the preoperative period, a conventional clinical assessment including routine physical examination, medical history, medication history, chest radiograph, ECG, and laboratory assessment of cardiovascular risk factors was performed in all patients and NT-proBNP concentration also was measured. The 12-lead ECGs were recorded after patients had rested for 1 minute in the supine position in an air-conditioned room.

Surgical risk was estimated according to the ASA classification. When NT-proBNP levels were greater than 
the normal range ( $>125 \mathrm{pg} / \mathrm{mL}$ ) or conventional clinical assessments were abnormal, we had cardiologists evaluate the need for additional tests including an echocardiogram, and perform preoperative assessment again as well as postoperative followup. Furthermore, we consulted anesthesiologists for cardiac care during surgery. Among the 328 patients, 42 patients (12.8\%) underwent transthoracic echocardiography based on a cardiologist's decision, and left ventricular ejection fraction was determined from Bmode images by using the Teichholz method, as described by Pombo et al. [15].

\section{Measurement of Serum NT-proBNP Concentrations}

For measurement of NT-proBNP, blood samples were taken from a peripheral vein. Serum samples were prepared within 30 minutes of collection by precooled centrifugation, and the serum NT-proBNP concentration was measured using an electrochemiluminescent immunoassay (ECLIA) kit (Roche Diagnostics, Tokyo, Japan) on an Elecsys ${ }^{\circledR} 2010$ analyzer (Roche). Blood was drawn on the day of or up to 48 hours before surgery. Preoperative NTproBNP level was known in real time, and investigators were not blinded to these results.

\section{Variables, Outcome Measures, Data Sources, and Bias}

Patient characteristics and medical and demographic details were obtained by reviewing medical documentation. Serial determination of myocardial necrosis markers and echocardiography also were performed for patients with chest pain, shortness of breath, sudden hemodynamic changes, or supraventricular or ventricular arrhythmias during the postoperative period. Cardiac complications and outcomes were considered to have occurred if they were documented in medical records. Medical records were documented as part of routine care; evaluation of those records for purposes of this study was performed by one of the authors (HU).

Cardiac complications were defined as congestive heart failure, major arrhythmia, acute myocardial infarction, or cardiac death. Congestive heart failure was diagnosed by means of clinical examination and chest radiography. Cardiac arrhythmia was defined as sustained ventricular tachycardia, new onset or in rapid response to atrial fibrillation. Acute myocardial infarction was defined as an elevation of myocardial necrosis markers by two times above the upper limits of the normal range in association with suggestive symptoms or new Q waves on the ECG. Cardiac death was defined as death from cardiac causes during the hospitalization. The endpoint of the study was the composite rate of 14-day postoperative cardiac complications. The first event was considered an outcome measure, with no double counting of events for the same patient.

\section{Statistical Analysis, Study Size}

Categorical variables were expressed as an absolute number and percentage and analyzed by the chi-square test or Fisher's exact test as appropriate. Continuous variables with normal distribution were expressed as mean $\pm \mathrm{SD}$ and analyzed by using the unpaired t-test, whereas age, body weight, creatinine clearance, NT-proBNP values, and length of surgery, which were not normally distributed, were expressed as median and interquartile range and analyzed by the Mann-Whitney U test. Probability values less than 0.01 were considered significant. Age, gender, body weight, laboratory data, ASA classification score, preoperative complications, current medication, cardiac examinations, and surgical factors were used as independent variables (Table 1).

NT-proBNP levels were dichotomized according to the best cutoff value established from receiver operating characteristic (ROC) curve analysis. Serum NT-proBNP levels are known to be critically affected by numerous factors including kidney function [17], age [8], gender, and body weight [6]. In the univariate analysis, age, gender, body weight, and variables with a probability less than 0.01 (Table 2) were included in the multivariate logistic regression model to determine the variables independently related to the postoperative outcome measures. Statistical analysis was conducted using SPSS Version 23.0 (IBM, Armonk, NY, USA) and Excel ${ }^{\circledR} 2013$ (Microsoft Corporation, Redmond, WA, USA).

\section{Baseline Characteristics}

An abnormal resting ECG was found in 96 patients (29.3\%) (Table 1); in detail, ST-T segment or T wave abnormalities were observed in 67 (4\%), left bundle branch block in $12(3.7 \%)$, atrial fibrillation in $11(3.4 \%)$, Q wave suggestive of previous acute myocardial infarction in three $(0.9 \%)$, and frequent premature ventricular complexes in three $(0.9 \%)$. Postoperative cardiac complications were encountered in 24 patients (7.3\%); congestive heart failure in 16, major arrhythmia in three, acute myocardial infarction in four, and cardiac death in one. The retrospective comparison of the baseline characteristics of patients with and without cardiac events was presented (Table 2). Patients with cardiac events tended to have lower creatinine clearance, higher ASA scores, the presence of congestive heart failure, atrial fibrillation, use of warfarin, 
Table 1. Baseline characteristics of the overall population

\begin{tabular}{|c|c|}
\hline Characteristic & Values \\
\hline Age (years) & $83(77-88)$ \\
\hline \multicolumn{2}{|l|}{ Gender } \\
\hline Female & $263(80 \%)$ \\
\hline Male & $65(20 \%)$ \\
\hline Body weight (kg) & $43.6(38-50.4)$ \\
\hline \multicolumn{2}{|l|}{ Laboratory data } \\
\hline Serum creatinine (mg/dL) & $\begin{array}{l}0.63(0.53- \\
0.76)\end{array}$ \\
\hline Creatinine clearance (mL/minute) & $47.5(38-60)$ \\
\hline Hemoglobin (g/dL) & $11.6 \pm 1.7$ \\
\hline NT-proBNP (pg/mL) & $299(151-554)$ \\
\hline ASA classification score & $1.9 \pm 0.6$ \\
\hline \multicolumn{2}{|l|}{ Preoperative complications } \\
\hline Myocardial infarction & $15(4.6 \%)$ \\
\hline Angina pectoris & $11(3.4 \%)$ \\
\hline $\begin{array}{l}\text { Surgical or percutaneous myocardial } \\
\text { revascularization }\end{array}$ & $4(1.2 \%)$ \\
\hline Congestive heart failure & $18(5.5 \%)$ \\
\hline Hypertension & $183(55.8 \%)$ \\
\hline Diabetes mellitus & $65(19.8 \%)$ \\
\hline Hypercholesterolemia & $38(11.6 \%)$ \\
\hline Chronic obstructive pulmonary disease & $11(3.4 \%)$ \\
\hline Previous cerebrovascular event & $62(18.9 \%)$ \\
\hline Atrial fibrillation & $16(4.9 \%)$ \\
\hline \multicolumn{2}{|l|}{ Current medication } \\
\hline Beta-blocker & $19(5.8 \%)$ \\
\hline $\begin{array}{l}\text { Angiotensin-converting enzyme inhibitor or } \\
\text { angiotensin receptor blocker }\end{array}$ & $91(27.7 \%)$ \\
\hline Diuretic & $40(12.2 \%)$ \\
\hline Statin & $56(17.1 \%)$ \\
\hline Antiplatelet & $82(25.0 \%)$ \\
\hline Warfarin & $13(4.0 \%)$ \\
\hline Ca channel blocker & $141(43.0 \%)$ \\
\hline Nitrate & $9(2.7 \%)$ \\
\hline \multicolumn{2}{|l|}{ Cardiac examination } \\
\hline Abnormal resting ECG & $96(29.3 \%)$ \\
\hline Enlarged heart on chest radiography* & $267(81.4 \%)$ \\
\hline $\begin{array}{l}\text { Left ventricular ejection fraction on } \\
\text { echocardiography }(\%)^{* *}\end{array}$ & $65.2 \pm 8.8$ \\
\hline \multicolumn{2}{|l|}{ Surgical factor } \\
\hline Length of surgery (minutes) & $32(22-50)$ \\
\hline Femoral neck fracture & $159(48.5 \%)$ \\
\hline Bipolar hip arthroplasty & $100(30.5 \%)$ \\
\hline General anesthesia & $115(35.1 \%)$ \\
\hline
\end{tabular}

Values expressed as mean $\pm \mathrm{SD}$, median (interquartile range), or number (percentage); ASA = American Society of Anesthesiologists; NT-proBNP $=\mathrm{N}$-terminal pro-B type natriuretic peptide; $*$ cardiothoracic ratio $>0.5$; **available for 42 patients. and abnormal resting ECG, and these were considered in our multivariate model.

\section{Results}

The median level of preoperative NT-proBNP was higher in patients with postoperative complications than in those without (1090 [interquartile range, $614-3191 \mathrm{pg} / \mathrm{mL}$ ] vs $283 \mathrm{pg} / \mathrm{mL}$ [137-507 pg/mL], $\mathrm{p}<0.001)$. After controlling for relevant confounding variables including age, gender, body weight, and creatinine clearance, we found that preoperative NT-proBNP greater than $600 \mathrm{pg} / \mathrm{mL}$ (odds ratio [OR], 12.902; 95\% CI, 4.389-37.926; $\mathrm{p}<0.001)$ and ASA classification (OR, 5.368; 95\% CI, 2.214-13.630; $\mathrm{p}<0.001)$ were independently associated with postoperative cardiac complications. We identified no other factors that were independently associated with postoperative cardiac events (Table 3). The ROC curve analysis (Fig. 1) indicated that the best cutoff level of NTproBNP for predicting postoperative cardiac complications was $600 \mathrm{pg} / \mathrm{mL}$, with sensitivity, specificity, positive predictive value, and negative predictive value of $79 \%, 81 \%, 25 \%$, and $98 \%$, respectively. The area under the ROC curve (AUC) was 0.87 ( $\mathrm{p}<0.001 ; 95 \% \mathrm{CI}$, 0.796-0.936).

\section{Discussion}

Elderly patients undergoing hip fracture surgery are at risk for cardiac complications, which are not consistently predicted by conventional preoperative clinical assessments. As these complications adversely affect the short-term prognosis during the postoperative period, it is desirable to identify patients at risk for postoperative cardiac complications more easily, accurately, and less expensively $[2,12,16]$. There are various available tools for identification of risk for postoperative cardiac complications other than ASA classification, including the AHA guidelines, GCRI, and RCRI. However, those tools consist of known cardiac risk factors including medical histories or physical examinations. Even when no abnormalities were detected on routine preoperative assessments, some patients experience postoperative cardiac events. Others have suggested that preoperative NT-proBNP is associated with postoperative cardiac complications in patients with hip fractures $[12,14]$, but those studies included patients with renal dysfunction. As NT-proBNP values are known to be influenced by renal function [17], we sought to assess more accurately the association between preoperative NT- 
Table 2. Retrospective comparison of baseline characteristics between patients with and without cardiac events

\begin{tabular}{|c|c|c|c|}
\hline Variable & $\begin{array}{l}\text { With cardiac events } \\
\mathrm{n}=24\end{array}$ & $\begin{array}{l}\text { Without cardiac events } \\
\mathrm{n}=304\end{array}$ & $\mathrm{p}$ Value \\
\hline Age (years) & $85.5(81-90)$ & $83(77-88)$ & 0.163 \\
\hline Gender (male/female) & $4 / 20$ & $61 / 243$ & 0.464 \\
\hline Body weight (kg) & $45(37-48.3)$ & $43.4(38-50.7)$ & 0.363 \\
\hline \multicolumn{4}{|l|}{ Laboratory data } \\
\hline Serum creatinine $(\mathrm{mg} / \mathrm{dL})$ & $0.74 \pm 0.23$ & $0.66 \pm 0.18$ & 0.035 \\
\hline Creatinine clearance (mL/minute) & $37(34-46)$ & $48(39-60)$ & 0.001 \\
\hline Hemoglobin (g/dL) & $11.4 \pm 2.1$ & $11.6 \pm 1.7$ & 0.539 \\
\hline NT-proBNP (pg/mL) & $1090(633-3101)$ & $283(138-504)$ & $<0.001$ \\
\hline ASA classification score & $2.4 \pm 0.5$ & $1.8 \pm 0.5$ & $<0.001$ \\
\hline \multicolumn{4}{|l|}{ Preoperative complications } \\
\hline Myocardial infarction & $2(8.3 \%)$ & $13(4.3 \%)$ & 0.301 \\
\hline Angina pectoris & $1(4.2 \%)$ & $10(3.3 \%)$ & 0.572 \\
\hline Surgical or percutaneous myocardial revascularization & $1(4.2 \%)$ & $3(1.0 \%)$ & 0.263 \\
\hline Congestive heart failure & $5(20.8 \%)$ & $13(4.3 \%)$ & 0.006 \\
\hline Hypertension & $12(50.0 \%)$ & $171(56.3 \%)$ & 0.553 \\
\hline Diabetes mellitus & $6(25.0 \%)$ & $59(19.4 \%)$ & 0.318 \\
\hline Hypercholesterolemia & $2(8.3 \%)$ & $36(11.8 \%)$ & 0.456 \\
\hline Chronic obstructive pulmonary disease & $0(0.0 \%)$ & $11(3.6 \%)$ & 0.428 \\
\hline Previous cerebrovascular event & $6(25.0 \%)$ & $56(18.4 \%)$ & 0.289 \\
\hline Atrial fibrillation & $5(20.8 \%)$ & $11(3.6 \%)$ & 0.003 \\
\hline \multicolumn{4}{|l|}{ Current medication } \\
\hline Beta-blocker & $4(16.7 \%)$ & $15(4.9 \%)$ & 0.041 \\
\hline $\begin{array}{l}\text { Angiotensin-converting enzyme inhibitor or angiotensin receptor } \\
\text { blocker }\end{array}$ & $7(29.2 \%)$ & $84(27.6 \%)$ & 0.872 \\
\hline Diuretic & $6(25.0 \%)$ & $34(11.2 \%)$ & 0.056 \\
\hline Statin & $3(12.5 \%)$ & $53(17.4 \%)$ & 0.388 \\
\hline Antiplatelet & $10(41.7 \%)$ & $72(23.7 \%)$ & 0.050 \\
\hline Warfarin & $5(20.8 \%)$ & $8(2.6 \%)$ & 0.001 \\
\hline Ca channel blocker & $9(37.5 \%)$ & $132(43.4 \%)$ & 0.573 \\
\hline Nitrate & $0(0.0 \%)$ & $9(3.0 \%)$ & 0.500 \\
\hline \multicolumn{4}{|l|}{ Cardiac examination } \\
\hline Abnormal resting ECG & $14(58.3 \%)$ & $82(27.0 \%)$ & 0.001 \\
\hline Enlarged heart on chest radiography & $21(87.5 \%)$ & $246(80.9 \%)$ & 0.314 \\
\hline Left ventricular ejection fraction on echocardiography $(\%) *$ & $59.3 \pm 8.8$ & $67.0 \pm 8.1$ & 0.043 \\
\hline \multicolumn{4}{|l|}{ Surgical factor } \\
\hline Length of surgery (minutes) & $30(21-47)$ & $32(23-50)$ & 0.767 \\
\hline Femoral neck fracture & $13(54.2 \%)$ & $146(48.0 \%)$ & 0.562 \\
\hline Bipolar hip arthroplasty & $9(37.5 \%)$ & $91(29.9 \%)$ & 0.438 \\
\hline General anesthesia & $14(58.3 \%)$ & $101(33.2 \%)$ & 0.013 \\
\hline
\end{tabular}

Values expressed as mean $\pm \mathrm{SD}$, median (interquartile range), or number (percentage); $\mathrm{p}$ values were calculated by unpaired t test, MannWhitney U test, chi-square test, or Fisher's exact test; NT-proBNP $=$ N-terminal pro-B type natriuretic peptide; ASA = American Society of Anesthesiologists; *available for 42 patients.

proBNP and cardiac complications after hip fracture surgery by excluding patients with renal dysfunction.

This study had several limitations. First, blinded NTproBNP measurements were not performed because of the nature of our study. When preoperative NT-proBNP levels were greater than the normal range, we consulted cardiologists for further evaluations, or contacted anesthesiologists to discuss anesthesia during the operation. The second limitation was that only the ASA classification was evaluated as the preoperative risk 
Table 3. Multivariate logistic regression analysis of adjusted risk factors related to postoperative cardiac outcome measures

\begin{tabular}{lccc}
\hline Variable & Odds ratio & p Value & $95 \%$ CI \\
\hline NT-proBNP $>600 \mathrm{pg} / \mathrm{mL}$ (yes/no) & 12.9 & $<0.001$ & $4.4-37.9$ \\
ASA classification* & 5.4 & $<0.001$ & $2.1-13.6$
\end{tabular}

$\mathrm{NT}$-proBNP $=\mathrm{N}$-terminal pro-B type natriuretic peptide; ASA = American Society of Anesthesiologists; *the odds ratio increase for ASA classification is presented for each grade; in other words, the odds ratio increases 5.4-fold for each ASA grade increase (from ASA 1 to 2, from 2 to 3 , etc).

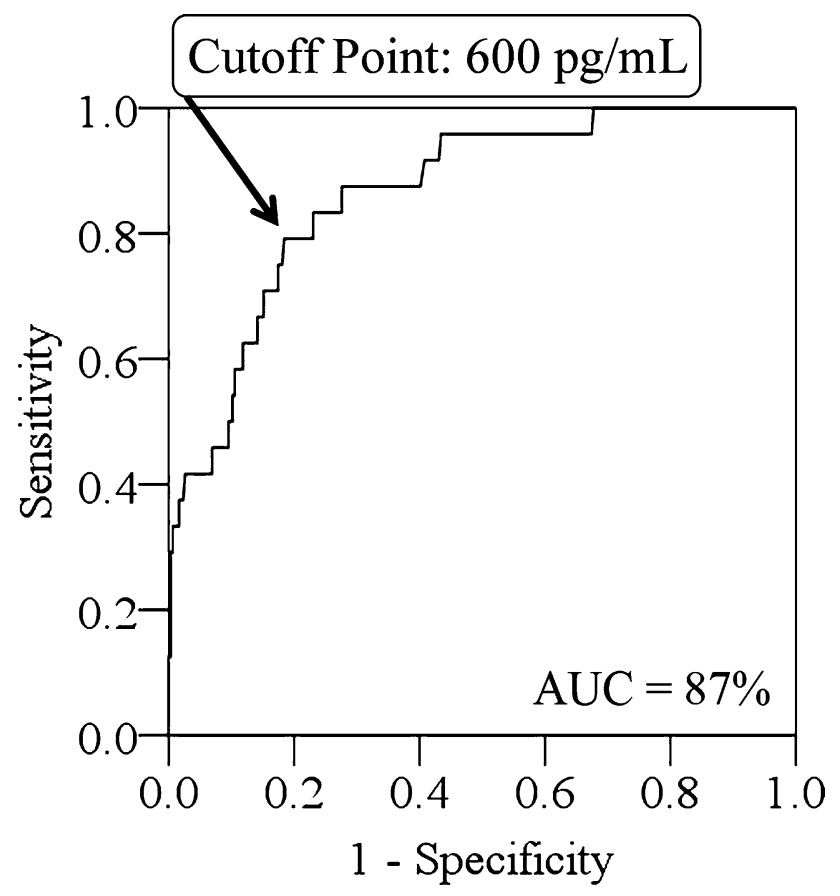

Fig. 1 The NT-proBNP for prediction of cardiac outcome measures using ROC curve analysis is shown. The value of $600 \mathrm{pg} / \mathrm{mL}$ represents the cutoff point of NT-proBNP with the best sensitivity and specificity. NT-proBNP $=\mathrm{N}$-terminal pro-brain type natriuretic peptide; $\mathrm{ROC}=$ receiver operating characteristic; $\mathrm{AUC}=$ area under the curve.

assessment tool for physical status. The ASA classification score was shown to be a useful predictor of postoperative complications; however, its subjectivity results in considerable interobserver variation and disagreement $[1,11]$. The GCRI and RCRI might be more useful tools to assess cardiac function than the ASA classification, but both of those indices are influenced by renal function. Because our study excluded patients with renal dysfunction, we believe that neither of these indices was suitable for this study. Third, only preoperative NT-proBNP levels were assessed in the study. Left ventricular dysfunction or cardiac failure might have developed postoperatively in some patients despite preoperative cardiac function being normal. Chong et al. [3] reported that preoperative and/or postoperative NT-proBNP could be used as a perioperative screening marker either to identify patients at high risk for postoperative cardiac complications or to continue to guide management. A comparison between preoperative and postoperative NT-proBNP levels might be more useful for clarifying the onset of complications. The fourth limitation was that the patients with renal dysfunction (111 of 439; $25.3 \%$ ) were excluded in our study, although some elderly patients with hip fractures actually do have impaired renal function. However, future studies should include broader patient populations to validate the use of NT-proBNP as a risk factor in patients with renal dysfunction. The fifth was that echocardiography was performed in only 42 patients, limiting the comparison of NT-proBNP with echocardiographic results. Whether it was performed was at the cardiologist's discretion, and the cardiologist did have access to the NT-proBNP values. However, the main objective of our study was to compare the performance of NT-proBNP with the routine preoperative examinations, medical history, and physical examination. Finally, our study did not deal explicitly with cost-effectiveness. That said, the NT-proBNP test costs USD 13 (Japanese yen [JPY] 1400) at our center, compared with USD 80 (JPY 8800 ) for an echocardiogram, therefore the cost for the NTproBNP does not seem to be a large expense considering its potential advantages.

The utility of NT-proBNP in anticipating postoperative cardiac complications has been reported by others in orthopaedic populations $[3,12]$. According to Chong et al. [3], the cutoff level of NT-proBNP $(842 \mathrm{pg} / \mathrm{mL})$ had a sensitivity of $90 \%$ and specificity of $74 \%$ for postoperative cardiac complications after emergency lower-limb orthopaedic surgery. Their study population was similar to ours, except they included patients with renal dysfunction, which likely explained the higher NT-proBNP cutoff point they obtained. According to Nordling et al. [12], an elevated perioperative NT-proBNP level is common in patients with hip fracture, and it is an independent predictor of shortterm and long-term mortality superior to the commonly used clinical risk scores. Although their study was conducted for a longer period than our study, it had several limitations. First, their study had smaller numbers of patients $(n=182)$ compared with our study $(n=328)$, and they included patients without preoperative NTproBNP values, which might result in elevated cutoff values from surgical stress. Although NT-proBNP is 
strongly affected by kidney function, their study did not include an index of renal function such as serum creatinine or creatinine clearance. In their study, 11 patients $(6 \%)$ died during the first 30 days after hospitalization among a total of 182 patients. Only one of 328 patients $(0.3 \%)$ in our study died within 14 days. Early interventions by cardiologists or anesthesiologists in the case of high preoperative levels of NT-proBNP may have helped to decrease the early mortality in our study patients.

In our study, eight of 24 patients with postoperative cardiac complications did not have preoperative cardiac risk factors such as a history of congestive heart failure, atrial fibrillation, current beta-blocker or warfarin use, or an abnormal ECG (Table 2). However, the mean NTproBNP levels for those eight patients was $1028 \mathrm{pg} / \mathrm{mL}$; our results suggest that NT-proBNP greater than $600 \mathrm{pg} /$ $\mathrm{mL}$ was sensitive and specific for anticipating postoperative cardiac complications. We believe that when NTproBNP is greater than $600 \mathrm{pg} / \mathrm{mL}$ even in patients without preoperative known cardiac risks, it is advisable to consult cardiologists and/or anesthesiologists, and to obtain informed consent from patients' families regarding to possible postoperative cardiac complications. Our findings suggest that NT-proBNP could help to identify patients who might need additional tests such as echocardiography for more precise evaluation of the postoperative cardiac risk.

Preoperative NT-proBNP greater than $600 \mathrm{pg} / \mathrm{mL}$ is independently associated with an increased risk of postoperative cardiac complications in patients with hip fractures who do not have renal dysfunction. Measurement of NTproBNP in addition to conventional preoperative cardiac examinations may help anticipate postoperative cardiac complications after hip fracture surgery. Future studies might develop a simple index for prediction of postoperative cardiac complications including more-refined cutoff values of NT-proBNP, especially in patients with no previous cardiovascular history and who take no medications.

\section{References}

1. Castillo J, Canet J, Gomar C, Hervás C. [Imprecise status allocation by users of the American Society of Anesthesiologists classification system: survey of Catalan anesthesiologists][in Spanish]. Rev Esp Anestesiol Reanim. 2007;54:394-398.

2. Choi JH, Cho DK, Song YB, Hahn JY, Choi S, Gwon HC, Kim DK, Lee SH, Oh JK, Jeon ES. Preoperative NT-proBNP and CRP predict perioperative major cardiovascular events in non-cardiac surgery. Heart. 2010;96:56-62.

3. Chong CP, Ryan JE, van Gaal WJ, Lam QT, Sinnappu RN, Burrell LM, Savige J, Lim WK. Usefulness of N-terminal probrain natriuretic peptide to predict postoperative cardiac complications and long-term mortality after emergency lower limb orthopedic surgery. Am J Cardiol. 2010;106:865-872.
4. Cockcroft DW, Gault MH. Prediction of creatinine clearance from serum creatinine. Nephron. 1976;16:31-41.

5. Eagle KA, Brundage BH, Chaitman BR, Ewy GA, Fleisher LA, Hertzer NR, Leppo JA, Ryan T, Schlant RC, Spencer WH 3rd, Spittell JA Jr, Twiss RD, Ritchie JL, Cheitlin MD, Gardner TJ, Garson A Jr, Lewis RP, Gibbons RJ, O'Rourke RA, Ryan TJ. Guidelines for perioperative cardiovascular evaluation for noncardiac surgery: report of the American College of Cardiology/ American Heart Association Task Force on Practice Guidelines. Committee on Perioperative Cardiovascular Evaluation for Noncardiac Surgery. Circulation. 1996;93:1278-1317.

6. Forfia PR, Watkins SP, Rame JE, Stewart KJ, Shapiro EP. Relationship between B-type natriuretic peptides and pulmonary capillary wedge pressure in the intensive care unit. J Am Coll Cardiol. 2005;45:1667-1671.

7. Goldman L, Caldera DL, Nussbaum SR, Southwick FS, Krogstad D, Murray B, Burke DS, O'Malley TA, Goroll AH, Caplan CH, Nolan J, Carabello B, Slater EE. Multifactorial index of cardiac risk in noncardiac surgical procedures. N Engl J Med. 1977;297:845-850.

8. Januzzi JL, van Kimmenade R, Lainchbury J, Bayes-Genis A, Ordonez-Llanos J, Santalo-Bel M, Pinto YM, Richards M. NTproBNP testing for diagnosis and short-term prognosis in acute destabilized heart failure: an international pooled analysis of 1256 patients: the International Collaborative of NT-proBNP Study. Eur Heart J. 2006;27:330-337.

9. Kadono Y, Yasunaga H, Horiguchi H, Hashimoto H, Matsuda S, Tanaka S, Nakamura K. Statistics for orthopedic surgery 20062007: data from the Japanese Diagnosis Procedure Combination database. J Orthop Sci. 2010;15:162-170.

10. Lee TH, Marcantonio ER, Mangione CM, Thomas EJ, Polanczyk CA, Cook EF, Sugarbaker DJ, Donaldson MC, Poss R, Ho KK, Ludwig LE, Pedan A, Goldman L. Derivation and prospective validation of a simple index for prediction of cardiac risk of major noncardiac surgery. Circulation. 1999;100:1043-1049.

11. Mak PH, Campbell RC, Irwin MG; American Society of Anesthesiologists. The ASA Physical Status Classification: interobserver consistency. American Society of Anesthesiologists. Anaesth Intensive Care. 2002;30:633-640.

12. Nordling P, Kiviniemi T, Strandberg M, Strandberg N, Airaksinen J. Predicting the outcome of hip fracture patients by using Nterminal fragment of pro-B-type natriuretic peptide. BMJ Open. 2016;6:e009416.

13. Orimo H, Yaegashi Y, Onoda T, Fukushima Y, Hosoi T, Sakata K. Hip fracture incidence in Japan: estimates of new patients in 2007 and 20-year trends. Arch Osteoporos. 2009;4:71-77.

14. Oscarsson A, Fredrikson M, Sörliden M, Anskär S, Eintrei C. Nterminal fragment of pro-B-type natriuretic peptide is a predictor of cardiac events in high-risk patients undergoing acute hip fracture surgery. Br J Anaesth. 2009;103:206-212.

15. Pombo JF, Troy BL, Russell RO Jr. Left ventricular volumes and ejection fraction by echocardiography. Circulation. 1971;43:480 490.

16. Ryding AD, Kumar S, Worthington AM, Burgess D. Prognostic value of brain natriuretic peptide in noncardiac surgery: a metaanalysis. Anesthesiology. 2009;111:311-319.

17. Takase H, Dohi Y. Kidney function crucially affects B-type natriuretic peptide (BNP), N-terminal proBNP and their relationship. Eur J Clin Invest. 2014;44:303-308.

18. Vacanti CJ, VanHouten RJ, Hill RC. A statistical analysis of the relationship of physical status to postoperative mortality in 68,388 cases. Anesth Analg. 1970;49:564-566.

19. Villacorta Junior H, Castro IS, Godinho M, Mattos C, Visconti R, Saud M, Novais M, Murad C, Nogueira F. B-type natriuretic peptide is predictive of postoperative events in orthopedic surgery. Arq Bras Cardiol. 2010;95:743-748. 